10,11

\title{
Энергия фононов и нулевых колебаний в сжатых кристаллических инертных газах
}

\author{
(C) Е.П. Троицкая ${ }^{1}$, Е.А. Пилипенко ${ }^{1}$, Е.Е. Горбенко ${ }^{2,3}$ \\ ${ }^{1}$ Донецкий фризико-технический институт им. А.А. Галкина, \\ Донецк, Украина \\ 2 Луганский национальный университет им. Тараса Шевченко, \\ Луганск, Украина \\ 3 Луганский национальный аграрный университет, \\ Луганск, Украина \\ E-mail: pilipenko.katerina@mail.ru
}

Поступила в Редакцию 24 апреля 2019 г.

В окончательной редакции 24 апреля 2019 г.

Принята к публикации 26 апреля 2019 г.

\begin{abstract}
В модели деформируемых и поряризуемых атомов построена динамическая матрица кристаллов инертных газов на основе неэмпирического короткодействующего потенциала отталкивания с учетом трехчастичного взаимодействия и деформации электронных оболочек атомов дипольного типа в парном и трехчастичном приближениях. Проведены ab initio расчеты энергии фононов для сжатых кристаллов инертных газов в двух и десяти точках главного значения метода Чади-Коэна в широком интервале давлений. Показано, что в фононных частотах вклад трехчастичных сил за счет перекрытия электронных оболочек соседних атомов мал на фоне парного взаимодействия даже при высоком давлении и наиболее заметен в Хе. Вклад деформации электронных оболочек в парном и трехчастичном приближениях различен для разных точек главного значения и увеличивается с ростом давления. Проведено сравнение рассчитанной энергии нулевых колебаний методом Чади-Коэна в кристаллах ряда $\mathrm{Ne}-\mathrm{Xe} \mathrm{c} \mathrm{имеющимися} \mathrm{экспериментальными} \mathrm{данными}$ при $p=0$ и расчетами других авторов.
\end{abstract}

Ключевые слова: кристаллы инертных газов, трехчастичное взаимодействие, деформация электронных оболочек, фононные частоты, энергия нулевых колебаний, высокое давление.

DOI: 10.21883/FTT.2019.10.48266.468

\section{1. Введение}

Кристаллы инертных газов (КИГ) являются простейшими молекулярными кристаллами, которые состоят из слабовзаимодействующих атомов с полностью заполненными оболочками. Как показывает эксперимент, все КИГ при нормальном давлении кристаллизуются в ГЦК структуру [1]. В отличие от более тяжелых КИГ, в которых под давлением происходят структурные ГЦК-ГПУ-переходы, кристаллический неон сохраняет ГЦК-структуру вплоть до давления металлизации. Однако в ранних теоретических работах [2,3] было показано, что при $p=0$ предпочтительной является гексагональная плотноупакованная (ГПУ) структура для всех КИГ. Такое несоответствие теории и эксперимента, известное как „проблема структуры КИГ“ [4,5], породило интенсивные дебаты об эффектах, обусловливающих разницу в предсказанных энергиях ГЦК- и ГПУ-решеток. Хотя эта разница на самом деле намного меньше $(\approx 0.01 \%$ энергии связи) [6], чем изначально предполагалось, она является решающей и стимулировала множество теоретических исследований межатомных взаимодействий КИГ.

Одним из наиболее известных первопринципных расчетов КИГ является теория функционала плотности (density functional theory - DFT). Только в некоторых из работ на основе DFT получена энергетическая выгодность ГЦК-структуры при нормальном давлении [7-9]. В работе [10] приведен обзор различных функционалов DFT и показано, что наибольшие ошибки наблюдаются при расчетах кристаллического Ne. Это связано с тем, что в теории функционала плотности используется модель жестких сферических атомов. Несмотря на то, что поляризуемость КИГ достаточно мала (для $\mathrm{Ne}$ она равна 2.67 a.u. [11]), эти кристаллы не могут быть адекватно описаны в рамках модели сферических атомов, поскольку основные сдерживающие их силы - дальнодействующие силы Ван-дер-Ваальса есть результат взаимного деформирующего и поляризующего действия атомов друг на друга. В настоящее время DFT не в состоянии учесть оба дисперсных типа взаимодействий таких как дальнодействующее взаимодействие Ван-дер-Ваальса (потенциал притяжения), так и эффекты перекрытия электронных оболочек атома в короткодействующем потенциале отталкивания. По мнению авторов $[12,13]$ квантово-теоретический метод обеспечивает описание обоих типов взаимодействия с достаточно высокой точностью. На основе этого метода в работах $[14,15]$ для $\mathrm{Ne}$ и $\mathrm{Ar}$ были получены близкие к эксперименту уравнения состояния (equation of state - 
EOS) в широком интервале давления с учетом двух-, трех- и четырехчастичных сил, а также ангармонического приближения для решеточных колебаний и температурных эффектов в модели Эйнштейна. Наиболее значительные поправки в $\mathrm{EOS} \mathrm{Ne}$ и $\mathrm{Ar}$ вносят энергия нулевых колебаний в гармоническом приближении при низких давлениях и многочастичное взаимодействие при высоких давлениях [14,15].

Авторы $a b$ initio теории связанных кластеров кристаллов инертных газов [16-18] при расчете энергии связи, показали, что энергия нулевых колебаний является основной причиной предпочтения ГЦК- перед ГПУ-структурой, за которой следует гораздо меньший вклад от короткодействующих трехчастичных сил.

В недавних работах $[19,20]$ формирование структуры твердого тела из атомов и молекул в газовой или жидкой фазе изучалось на основе потенциала Леннард-Джонса. Наиболее реалистичный расширенный потенциал Леннард-Джонса, выбранный на основе расчетов связанных кластеров для димера инертного газа, приводит к повышению числа неизоморфных кластеров, хотя потенциальная кривая при этом очень похожа на кривую $(6,12)$ потенциала Леннард-Джонса. Авторы придерживаются мнения, что между теорией и экспериментом имеется все еще большое расхождение, несмотря на все достижения в кластерной физике.

В связи с этим важно рассчитать энергию нулевых колебаний из первых принципов в едином подходе, позволяющем получить как многочастичное взаимодействие в короткодействующем потенциале отталкивания, так и дальнодействующее взаимодействие Ван-дер-Ваальса (потенциал притяжения), которое представляет собой результат взаимного деформирующего и поляризующего действия атомов друг на друга.

В настоящей работе на основе неэмпирической версии квантово-механической модели деформируемых и поляризуемых атомов (модель Толпыго см. [21] и ссылки там) построена динамическая матрица с учетом обоих видов трехчастичных взаимодействий как за счет перекрытия электронных оболочек, так и за счет их деформации. Это позволит рассчитать фононные частоты в любой точке зоны Бриллюэна и, используя метод Чади-Коэна, энергию нулевых колебаний в широком интервале давлений.

Цель настоящей работы - исследовать влияния трехчастичного взаимодействия в короткодействующем потенциале отталкивания и деформации электронных оболочек атомов дипольного типа в парном и трехчастичном приближении на фононы и энергию нулевых колебаний КИГ при низких и высоких давлениях.

\section{2. Динамическая матрица кристаллов инертных газов}

В используемой модели Толпыго потенциальная энергия решетки $U$ получена путем нахождения минимума среднего гамильтониана электронной подсистемы $\bar{H}$ по вариационным параметрам $c_{i}^{l}$ и $c_{i j}^{l l^{\prime}}$, описывающим слабую деформацию электронной волновой функции, при произвольных фиксированных смещениях ядер $\mathbf{u}^{l}$ и произвольных дипольных моментах $\mathbf{P}^{l}$ (см. [21] и ссылки там)

$$
\begin{aligned}
U=\min \bar{H}=\mathrm{const} & +\sum_{l}\left\{\frac{\left(\mathbf{P}^{l}\right)^{2}}{2 \alpha}+\boldsymbol{\beta}^{l} \cdot \mathbf{P}^{l}-\frac{1}{2} \sum_{l^{\prime}} \frac{C}{\left|\mathbf{r}^{l l^{\prime}}\right|^{6}}\right. \\
& \left.+\frac{1}{2} \sum_{l^{\prime}} K\left(\mathbf{P}^{l}, \mathbf{P}^{l^{\prime}}\right)+\frac{1}{2} \sum_{l^{\prime}}^{n . n .} U_{s r}\left(\left|\mathbf{r}^{l}-\mathbf{r}^{l^{\prime}}\right|\right)\right\}
\end{aligned}
$$

Первые два члена описывают деформацию электронных оболочек в дипольном приближении $(\alpha-$ коэффициент дипольной поляризуемости). Следующий член дает силы Ван-дер-Ваальса. $K$ - кулоновское (в классическом смысле) взаимодействие всех диполей $\mathbf{P}^{l}$ между собой. Последнее слагаемое в (1) представляет собой короткодействующие отталкивание $E_{s}$, которое содержит трехчастичное взаимодействие за счет деформации электронных оболочек атомов и их перекрытия [22].

Тогда уравнения колебаний запишутся как

$$
m \ddot{u}_{\alpha}^{l}=-\frac{\partial U}{\partial u_{\alpha}^{l}}, \quad \frac{\partial U}{\partial P_{\alpha}^{l}}=0 .
$$

Выполняя дифференцирование в уравнениях (2), подставляя все переменные $\mathbf{p}^{l}=e \mathbf{u}^{l}, \mathbf{P}^{l}$ в виде плоских волн $\exp \{i \mathbf{k r}-i \omega t\}$ и суммируя по $l^{\prime} l^{\prime \prime}$, можно получить уравнения для амплитуд $p_{\alpha}, P_{\alpha}$ с учетом трехчастичного взаимодействия.

Для определения собственных частот $\omega_{\lambda \mathbf{k}}$ две группы уравнений (2) удобнее записать как

$$
\begin{aligned}
& M \omega_{\lambda \mathbf{k}}^{2} p_{\alpha}=\sum_{\beta}\left(A_{\alpha \beta} p_{\beta}+B_{\alpha \beta} P_{\beta}\right), \\
& m \omega_{\lambda \mathbf{k}}^{2} P_{\alpha}=\sum_{\beta}\left(B_{\beta \alpha}^{*} p_{\beta}+C_{\alpha \beta} P_{\beta}\right),
\end{aligned}
$$

где $M$ - масса атома, $m$ - некоторая „фиктивная“ масса порядка массы электронной оболочки, введенная исключительно для удобства расчетов, так как диагонализация матрицы $6 \times 6$ технически более проста, чем процедура исключения всех $\mathbf{P}$ из второй группы уравнений при $m=0$, как этого требует адиабатическое приближение. Таким образом, удобно ввести некоторую матрицу

$$
D=\left(\begin{array}{ll}
D^{(1)} & D^{(2)} \\
D^{(2)} & D^{(3)}
\end{array}\right),
$$

каждый элемент, которой представляет собой матрицу $3 \times 3$ :

$$
D^{(1)}=\left(\begin{array}{lll}
A_{11}(\mathbf{k}) & A_{12}(\mathbf{k}) & A_{13}(\mathbf{k}) \\
A_{21}(\mathbf{k}) & A_{22}(\mathbf{k}) & A_{23}(\mathbf{k}) \\
A_{31}(\mathbf{k}) & A_{32}(\mathbf{k}) & A_{33}(\mathbf{k})
\end{array}\right),
$$


Аналогично для матриц $D^{(2)}\left(B_{\alpha \beta}(\mathbf{k})\right)$ и $D^{(3)}\left(C_{\alpha \beta}(\mathbf{k})\right)$. Для получения частоты нужной размерности, каждый элемент матрицы нужно умножить на размерный множитель $2 \lambda=\frac{e^{2}}{a^{3}}(e-$ заряд электрона). Тогда

$$
\begin{gathered}
A_{\alpha \beta}(\mathbf{k})=\frac{e^{2}}{a^{3}}\left[\begin{array}{c}
B \chi_{\alpha \beta}(\mathbf{k})+\delta_{\alpha \beta}\left(H \mu(\mathbf{k})+G v_{\alpha}(\mathbf{k})+F \xi(\mathbf{k})+\right. \\
\left.+E \xi_{\alpha}(\mathbf{k})+V_{t} \vartheta_{\alpha}(\mathbf{k})\right)+\left(1-\delta_{\alpha \beta}\right) G \tau_{\alpha \beta}(\mathbf{k})
\end{array}\right] \\
B_{\alpha \beta}(\mathbf{k})=\frac{e^{2}}{a^{3}}\left[\delta_{\alpha \beta}\left(h \mu(\mathbf{k})+g v_{\alpha}(\mathbf{k})\right)+\left(1-\delta_{\alpha \beta}\right) g \tau_{\alpha \beta}(\mathbf{k})\right] \\
C_{\alpha \beta}(\mathbf{k})=\frac{e^{2}}{a^{3}}\left[\delta_{\alpha \beta} A^{-1}-\varphi_{\alpha \beta}(\mathbf{k})\right] .
\end{gathered}
$$

Где $\delta_{\alpha \beta}-$ символ Кронекера;

$$
\begin{gathered}
\mu(\mathbf{k})=3-\frac{1}{2} \sum_{\gamma \neq \delta} \cos k_{\gamma} \cos k_{\delta}, \\
v_{\alpha}(\mathbf{k})=2-\cos k_{\alpha} \sum_{\gamma \neq \alpha} \cos k_{\gamma}, \quad \tau_{\alpha \beta}(\mathbf{k})=\sin k_{\alpha} \sin k_{\beta}, \\
\xi(\mathbf{k})=3-\sum_{\gamma} \cos 2 k_{\gamma}, \quad \zeta_{\alpha}(\mathbf{k})=1-\cos 2 k_{\alpha}, \\
\vartheta_{\alpha}(\mathbf{k})=1-\cos k_{\alpha+1} \cos k_{\alpha+2}, \quad \mathbf{k}=a \mathbf{K}=\pi \mathbf{q} .
\end{gathered}
$$

Здесь $H=H_{0}+\delta H, G=G_{0}+\delta G$, где $H_{0}(a \sqrt{2})$ и $G_{0}(a \sqrt{2})$ являются первой и второй производными короткодействующего парного потенциала отталкивания для равновесных расстояний первых соседей; аналогичные величин для вторых соседей $-F=H_{0}(2 a)$ и $E=G_{0}(2 a) ; B$ определяет взаимодействие Ван-дерВаальса; $h$ и $g$ - параметры деформации электронных оболочек атомов дипольного типа в парном приближении; k - безразмерный волновой вектор; $\chi_{\alpha \beta}(\mathbf{k})-$ функции k, происходящие от ван-дер-ваальсовских сил; $\varphi_{\alpha \beta}(\mathbf{k})$ - коэффициенты электрического поля, вызванного системой диполей $\mathbf{P}^{l} ; A-$ безразмерная поляризуемость атома. Параметры $\delta G, \delta H$ и $V_{t}$ описывают трехчастичные короткодействующие силы, обусловленные перекрытием электронных оболочек (недеформированных) атомов (см. [21] и ссылки там).

В работе [22] были рассмотрены трехчастичные силы, связанные с деформацией электронных оболочек атомов. В дальнейшем мы будем учитывать эти силы за счет переопределения параметров $g$ и $h$.

Для примера, приведем элементы матрицы $D^{(1)}$, в которой учли трехчастичные силы в короткодействующем потенциале отталкивания, обусловленные перекрытием электронных оболочек атомов. Диагональные элементы матрицы имеют вид

$$
\begin{aligned}
A_{11}(\mathbf{k})= & A_{x x}(\mathbf{k})=\frac{e^{2}}{a^{3}}\left[\left(H_{0}+\delta H\right) \mu(\mathbf{k})+\left(G_{0}+\delta G\right) v_{x}(\mathbf{k})\right. \\
& \left.+F \xi(\mathbf{k})+E \xi_{x}(\mathbf{k})+V_{t} \vartheta_{x}(\mathbf{k})+B \chi_{x x}(\mathbf{k})\right], \quad(10)
\end{aligned}
$$

\section{Недиагональные элементы}

$$
\begin{aligned}
& A_{12}(\mathbf{k})=A_{x y}(\mathbf{k})=\frac{e^{2}}{a^{3}}\left[\left(G_{0}+\delta G\right) \tau_{x y}(\mathbf{k})+B \chi_{x y}(\mathbf{k})\right], \\
& A_{13}(\mathbf{k})=A_{x z}(\mathbf{k})=\frac{e^{2}}{a^{3}}\left[\left(G_{0}+\delta G\right) \tau_{x z}(\mathbf{k})+B \chi_{x z}(\mathbf{k})\right] .
\end{aligned}
$$

Аналогично для остальных элементов матрицы, имея в виду, что $1 \rightarrow x, 2 \rightarrow y$ и $3 \rightarrow z, 4 \rightarrow x$ и т.д. Тогда, вклады трехчастичных сил в диагональные элементы матрицы приобретают вид

$$
\begin{aligned}
& A_{11}^{t}=A_{x x}^{t}=\frac{e^{2}}{a^{3}}\left[\delta H \mu(\mathbf{k})+\delta G v_{x}(\mathbf{k})+V_{t} \vartheta_{x}(\mathbf{k})\right], \\
& A_{22}^{t}=A_{y y}^{t}=\frac{e^{2}}{a^{3}}\left[\delta H \mu(\mathbf{k})+\delta G v_{y}(\mathbf{k})+V_{t} \vartheta_{y}(\mathbf{k})\right], \\
& A_{33}^{t}=A_{z z}^{t}=\frac{e^{2}}{a^{3}}\left[\delta H \mu(\mathbf{k})+\delta G v_{z}(\mathbf{k})+V_{t} \vartheta_{z}(\mathbf{k})\right] .
\end{aligned}
$$

В недиагональные

$$
\begin{aligned}
& A_{12}^{t}(\mathbf{k})=A_{21}^{t}(\mathbf{k})=A_{x y}^{t}(\mathbf{k})=\frac{e^{2}}{a^{3}} \delta G v_{x y}(\mathbf{k}), \\
& A_{13}^{t}(\mathbf{k})=A_{31}^{t}(\mathbf{k})=A_{x z}^{t}(\mathbf{k})=\frac{e^{2}}{a^{3}} \delta G v_{x z}(\mathbf{k}), \\
& A_{23}^{t}(\mathbf{k})=A_{32}^{t}(\mathbf{k})=A_{y z}^{t}(\mathbf{k})=\frac{e^{2}}{a^{3}} \delta G v_{y z}(\mathbf{k}) .
\end{aligned}
$$

Деформация электронных оболочек атомов дипольного типа в парном и трехчастичном приближениях описывается элементами матрицы $D^{(2)}$. Таким образом, диагонализация динамической матрицы $D$ даст нам фононные частоты в любой точке зоны Бриллюэна, например, в точках главного значения метода Чади-Коэна [23].

\section{3. Энергия фононов в точках главного значения}

Bсе параметры короткодействия как парные, так и терехчастичные рассчитываются точно [21]. При нахождении константы Ван-дер-Ваальса необходимо учитывать условие равновесия

$$
H_{0}+\delta H+2 F-2 R_{t}=0.30112 B,
$$

где $\quad R_{t}=-\frac{a}{6 e^{2}} \frac{d W_{3}(a)}{d a} ; \quad W_{3}(a)=-24 S^{2}(a \sqrt{2}) f\left(\frac{\sqrt{6}}{2} a\right)$, $S$ - интеграл перекрытия.

Для $\mathrm{Ne}$ в [24] приведены значения рассчитанных параметров парного короткодействия $G_{0}, H_{0}, E, F$, трехчастичного взаимодействия $\delta H, \delta G, V_{t}$, дипольной деформации $h, g$, а также параметр Ван-дер-Ваальса $B$ при различных сжатиях $u=\Delta V / V_{0}\left(\Delta V=V_{0}-V(p)\right.$, где $V_{0}$ - объем при $\left.p=0\right)$.

Суммы $\varphi_{\alpha \beta}(\mathbf{k})$ и $\chi_{\alpha \beta}(\mathbf{k})$ представляют собой вклад в динамическую матрицу дальнодействующих кулоновских и ван-дер-ваальсовых сил и рассчитываются точно. 
Таблица 1. Частоты $\hbar \omega_{\lambda \mathbf{k}}$ в моделях $M T_{0}$ и $M T_{2}$ и относительный вклад эффектов деформации электронных оболочек в парном и трехчастичном приближениях $\gamma_{1}$ при различных степенях сжатия $u$ для $\mathrm{Ne}$

\begin{tabular}{|c|c|c|c|c|c|c|c|c|c|c|c|c|}
\hline Теория & \multicolumn{4}{|c|}{$\hbar \omega_{\lambda \mathbf{k}}$ в модели $M T_{0}, \mathrm{meV}$} & \multicolumn{4}{|c|}{$\hbar \omega_{\lambda \mathbf{k}}$ в модели $M T_{2}, \mathrm{meV}$} & \multicolumn{4}{|c|}{$\gamma_{1}, \%$} \\
\hline$u$ & 0 & 0.3 & 0.7 & 0.76 & 0 & 0.3 & 0.7 & 0.76 & 0 & 0.3 & 0.7 & 0.76 \\
\hline & \multicolumn{12}{|c|}{$p, \mathrm{GPa}$} \\
\hline $\mathbf{k}, \lambda$ & 0 & 1.886 & 169.62 & 441.47 & 0 & 1.886 & 169.62 & 441.47 & 0 & 1.886 & 169.62 & 441.47 \\
\hline $\mathbf{k}_{1}[7 / 8 ; 3 / 8 ; 1 / 8]$ & $\begin{array}{l}4.53 \\
5.26 \\
6.06\end{array}$ & $\begin{array}{l}11.37 \\
13.71 \\
16.08\end{array}$ & $\begin{array}{l}65.11 \\
80.38 \\
95.71\end{array}$ & $\begin{array}{r}95.69 \\
118.13 \\
141.08\end{array}$ & $\begin{array}{l}4.53 \\
5.25 \\
6.04\end{array}$ & $\begin{array}{l}11.36 \\
13.65 \\
15.93\end{array}$ & $\begin{array}{l}64.73 \\
76.73 \\
78.19\end{array}$ & $\begin{array}{r}84.45 \\
95.01 \\
100.99\end{array}$ & $\begin{array}{l}0.03 \\
0.14 \\
0.28\end{array}$ & $\begin{array}{l}0.09 \\
0.48 \\
0.93\end{array}$ & $\begin{array}{r}0.59 \\
4.54 \\
18.30\end{array}$ & $\begin{array}{l}11.74 \\
19.56 \\
28.42\end{array}$ \\
\hline $\mathbf{k}_{2}[7 / 8 ; 1 / 8 ; 1 / 8]$ & $\begin{array}{l}4.42 \\
4.76 \\
6.39\end{array}$ & $\begin{array}{l}11.21 \\
12.21 \\
17.07\end{array}$ & $\begin{array}{c}63.92 \\
70.56 \\
102.07\end{array}$ & $\begin{array}{r}93.39 \\
103.39 \\
150.63\end{array}$ & $\begin{array}{l}4.42 \\
4.75 \\
6.37\end{array}$ & $\begin{array}{l}11.19 \\
12.18 \\
16.87\end{array}$ & $\begin{array}{l}63.40 \\
68.36 \\
79.05\end{array}$ & $\begin{array}{l}73.61 \\
92.78 \\
98.50\end{array}$ & $\begin{array}{l}0.04 \\
0.07 \\
0.35\end{array}$ & $\begin{array}{l}0.12 \\
0.24 \\
1.13\end{array}$ & $\begin{array}{r}0.81 \\
3.12 \\
22.56\end{array}$ & $\begin{array}{l}21.18 \\
10.27 \\
34.61\end{array}$ \\
\hline $\mathbf{k}_{3}[5 / 8 ; 5 / 8 ; 1 / 8]$ & $\begin{array}{l}4.03 \\
5.04 \\
6.21\end{array}$ & $\begin{array}{r}9.84 \\
13.15 \\
16.51\end{array}$ & $\begin{array}{l}55.33 \\
77.07 \\
98.81\end{array}$ & $\begin{array}{r}81.21 \\
113.19 \\
146.08\end{array}$ & $\begin{array}{l}4.03 \\
5.04 \\
6.19\end{array}$ & $\begin{array}{c}9.84 \\
13.10 \\
16.34\end{array}$ & $\begin{array}{l}55.01 \\
70.76 \\
79.61\end{array}$ & $\begin{array}{l}78.63 \\
80.03 \\
99.44\end{array}$ & $\begin{array}{l}0.00 \\
0.13 \\
0.30\end{array}$ & $\begin{array}{l}0.00 \\
0.45 \\
1.00\end{array}$ & $\begin{array}{r}0.57 \\
8.18 \\
19.43\end{array}$ & $\begin{array}{r}3.18 \\
29.30 \\
31.93\end{array}$ \\
\hline $\mathbf{k}_{4}[5 / 8 ; 3 / 8 ; 3 / 8]$ & $\begin{array}{l}3.69 \\
3.70 \\
6.58\end{array}$ & $\begin{array}{r}8.96 \\
9.84 \\
17.70\end{array}$ & $\begin{array}{c}50.00 \\
55.97 \\
106.88\end{array}$ & $\begin{array}{r}73.21 \\
82.19 \\
158.28\end{array}$ & $\begin{array}{l}3.69 \\
3.97 \\
6.55\end{array}$ & $\begin{array}{r}8.96 \\
9.84 \\
17.47\end{array}$ & $\begin{array}{l}49.49 \\
55.97 \\
78.55\end{array}$ & $\begin{array}{l}55.67 \\
69.69 \\
81.96\end{array}$ & $\begin{array}{l}0.00 \\
0.01 \\
0.41\end{array}$ & $\begin{array}{l}0.00 \\
0.03 \\
1.30\end{array}$ & $\begin{array}{r}1.03 \\
0.00 \\
26.51\end{array}$ & $\begin{array}{l}23.95 \\
15.20 \\
48.22\end{array}$ \\
\hline $\mathbf{k}_{5}[5 / 8 ; 3 / 8 ; 1 / 8]$ & $\begin{array}{l}3.99 \\
4.54 \\
6.58\end{array}$ & $\begin{array}{r}9.63 \\
11.47 \\
16.73\end{array}$ & $\begin{array}{l}53.32 \\
65.77 \\
98.68\end{array}$ & $\begin{array}{r}78.22 \\
96.21 \\
145.91\end{array}$ & $\begin{array}{l}3.99 \\
4.54 \\
6.56\end{array}$ & $\begin{array}{r}9.63 \\
11.44 \\
16.57\end{array}$ & $\begin{array}{l}53.08 \\
63.14 \\
80.01\end{array}$ & $\begin{array}{l}76.29 \\
82.38 \\
93.29\end{array}$ & $\begin{array}{l}0.02 \\
0.08 \\
0.25\end{array}$ & $\begin{array}{l}0.02 \\
0.26 \\
0.93\end{array}$ & $\begin{array}{r}0.45 \\
4.00 \\
18.92\end{array}$ & $\begin{array}{r}2.47 \\
14.37 \\
36.07\end{array}$ \\
\hline $\mathbf{k}_{6}[5 / 8 ; 1 / 8 ; 1 / 8]$ & $\begin{array}{l}3.73 \\
3.92 \\
5.76\end{array}$ & $\begin{array}{r}9.45 \\
9.99 \\
15.44\end{array}$ & $\begin{array}{l}53.66 \\
57.28 \\
93.14\end{array}$ & $\begin{array}{r}78.15 \\
83.75 \\
138.06\end{array}$ & $\begin{array}{l}3.73 \\
3.92 \\
5.75\end{array}$ & $\begin{array}{r}9.44 \\
9.98 \\
15.31\end{array}$ & $\begin{array}{l}53.38 \\
56.50 \\
78.39\end{array}$ & $\begin{array}{l}77.88 \\
81.41 \\
93.65\end{array}$ & $\begin{array}{l}0.02 \\
0.04 \\
0.27\end{array}$ & $\begin{array}{l}0.08 \\
0.13 \\
0.85\end{array}$ & $\begin{array}{r}0.51 \\
1.37 \\
15.84\end{array}$ & $\begin{array}{r}0.35 \\
2.79 \\
32.17\end{array}$ \\
\hline $\mathbf{k}_{6}[3 / 8 ; 3 / 8 ; 3 / 8]$ & $\begin{array}{l}3.25 \\
3.25 \\
6.21\end{array}$ & $\begin{array}{r}7.78 \\
7.78 \\
16.82\end{array}$ & $\begin{array}{c}43.05 \\
43.05 \\
102.02\end{array}$ & $\begin{array}{r}63.09 \\
63.09 \\
151.21\end{array}$ & $\begin{array}{l}3.25 \\
3.25 \\
6.18\end{array}$ & $\begin{array}{r}7.78 \\
7.78 \\
16.62\end{array}$ & $\begin{array}{l}42.11 \\
42.11 \\
77.46\end{array}$ & $\begin{array}{l}57.73 \\
57.73 \\
66.73\end{array}$ & $\begin{array}{l}0.00 \\
0.00 \\
0.39\end{array}$ & $\begin{array}{l}0.01 \\
0.01 \\
1.21\end{array}$ & $\begin{array}{r}2.19 \\
2.19 \\
24.07\end{array}$ & $\begin{array}{r}8.50 \\
8.50 \\
55.87\end{array}$ \\
\hline $\mathbf{k}_{8}[3 / 8 ; 3 / 8 ; 1 / 8]$ & $\begin{array}{l}2.98 \\
3.36 \\
5.36\end{array}$ & $\begin{array}{r}7.01 \\
8.57 \\
14.53\end{array}$ & $\begin{array}{l}38.74 \\
48.98 \\
88.14\end{array}$ & $\begin{array}{r}57.15 \\
71.36 \\
130.73\end{array}$ & $\begin{array}{l}2.98 \\
3.36 \\
5.35\end{array}$ & $\begin{array}{r}7.01 \\
8.57 \\
14.41\end{array}$ & $\begin{array}{l}37.36 \\
48.38 \\
74.31\end{array}$ & $\begin{array}{l}50.02 \\
69.62 \\
88.41\end{array}$ & $\begin{array}{l}0.00 \\
0.03 \\
0.27\end{array}$ & $\begin{array}{l}0.02 \\
0.10 \\
0.84\end{array}$ & $\begin{array}{r}3.57 \\
1.21 \\
15.70\end{array}$ & $\begin{array}{r}12.47 \\
2.43 \\
32.37\end{array}$ \\
\hline $\mathbf{k}_{9}[3 / 8 ; 1 / 8 ; 1 / 8]$ & $\begin{array}{l}2.57 \\
2.60 \\
4.33\end{array}$ & $\begin{array}{r}6.44 \\
6.46 \\
11.75\end{array}$ & $\begin{array}{l}36.31 \\
36.31 \\
71.59\end{array}$ & $\begin{array}{r}52.72 \\
53.13 \\
106.39\end{array}$ & $\begin{array}{l}2.57 \\
2.60 \\
4.32\end{array}$ & $\begin{array}{r}6.44 \\
6.45 \\
11.69\end{array}$ & $\begin{array}{l}36.19 \\
36.29 \\
65.12\end{array}$ & $\begin{array}{l}52.46 \\
52.72 \\
87.55\end{array}$ & $\begin{array}{l}0.01 \\
0.07 \\
0.16\end{array}$ & $\begin{array}{l}0.02 \\
0.03 \\
0.51\end{array}$ & $\begin{array}{l}0.33 \\
0.08 \\
9.04\end{array}$ & $\begin{array}{r}0.50 \\
0.77 \\
17.71\end{array}$ \\
\hline $\mathbf{k}_{10}[1 / 8 ; 1 / 8 ; 1 / 8]$ & $\begin{array}{l}1.35 \\
1.35 \\
2.48\end{array}$ & $\begin{array}{l}3.23 \\
3.23 \\
6.88\end{array}$ & $\begin{array}{l}17.84 \\
17.84 \\
42.12\end{array}$ & $\begin{array}{l}26.15 \\
26.15 \\
62.47\end{array}$ & $\begin{array}{l}1.35 \\
1.35 \\
2.48\end{array}$ & $\begin{array}{l}3.23 \\
3.23 \\
6.87\end{array}$ & $\begin{array}{l}17.78 \\
17.78 \\
40.61\end{array}$ & $\begin{array}{l}25.76 \\
25.76 \\
58.14\end{array}$ & $\begin{array}{l}0.00 \\
0.00 \\
0.07\end{array}$ & $\begin{array}{l}0.00 \\
0.00 \\
0.21\end{array}$ & $\begin{array}{l}0.38 \\
0.38 \\
3.60\end{array}$ & $\begin{array}{l}1.47 \\
1.47 \\
6.92\end{array}$ \\
\hline Сред.знач. & & & & & & & & & 0.15 & 0.37 & 6.98 & 17.16 \\
\hline
\end{tabular}

Примечание. $\gamma_{1}=\left[\left|\omega\left(M T_{0}\right)-\omega\left(M T_{2}\right)\right| / \omega\left(M T_{0}\right)\right] \cdot 100 \%$.

Они не зависят от конкретных параметров кристалла и одинаковы для всех веществ, имеющих одинаковый тип решетки. Точно рассчитанные значения сумм $\chi_{\alpha \beta}(\mathbf{k})$ и $\varphi_{\alpha \beta}(\mathbf{k})$ для двух и десяти точек главного значения приведены в [25].

В табл. 1 представлены фононные частоты $\hbar \omega_{\lambda}\left(\mathbf{k}_{i}\right)$ для $\mathrm{Ne}$, рассчитанные для десяти точек главного значения метода Чади-Коэна в моделях $M T_{0}$ (учитывается трехчастичное взаимодействие за счет перекрытия электронных оболочек и не учитывается деформация электронных оболочек атома) и $M T_{2}$ (учитываются трехчастичные силы за счет перекрытия электронных оболочек и деформация электронных оболочек атомов дипольного типа в парном и трехчастичном приближениях) при сжатиях $u$ от 0 до 0.76. Кроме этого, в табл. 1 показан относительный вклад деформации электронных оболочек $\gamma_{1}$. Как видно, фононные частоты достаточно чувствительны к добавлению этого вклада в парном и трехчастичном приближениях. Он варьируется в зависимости от точки зоны Бриллюэна от 0.35 до $55.87 \%$ при сжатии $u=0.76$, однако его средняя величина составляет 17\%. Аналогичная тенденция наблюдается 
Таблица 2. Энергия нулевых колебаний $E_{\text {zр }}$, рассчитанная по двухточечной схеме Чади-Коэна в моделях $M 3, M 3 a, M T_{0}, M T_{2}$ и относительные вклады трехчастичного взаимодействия и эффектов деформации электронных оболочек в парном и трехчастичном приближениях $\gamma_{i}$ при различных степенях сжатия для ряда $\mathrm{Ne}-\mathrm{Xe}$

\begin{tabular}{|c|c|c|c|c|c|c|c|}
\hline \multirow{2}{*}{$u$} & \multicolumn{4}{|c|}{$E_{\mathrm{zp}}, \mathrm{K}$} & \multirow{2}{*}{$\gamma_{1}, \%$} & \multirow{2}{*}{$\gamma_{2}, \%$} & \multirow{2}{*}{$\gamma_{3}, \%$} \\
\hline & $M T_{0}$ & $M T_{2}$ & M3 & $M 3 a$ & & & \\
\hline \multicolumn{8}{|c|}{$\mathrm{Ne}$} \\
\hline $\begin{array}{l}0 \\
0.3 \\
0.6 \\
0.7 \\
0.76\end{array}$ & $\begin{array}{c}79.309 \\
205.261 \\
695.224 \\
1200.4 \\
1767.08\end{array}$ & $\begin{array}{r}79.185 \\
204.203 \\
669.079 \\
1081.43 \\
1386.29\end{array}$ & $\begin{array}{r}79.457 \\
205.473 \\
696.367 \\
1203.27 \\
1771.79\end{array}$ & $\begin{array}{r}79.356 \\
204.597 \\
674.852 \\
1106.64 \\
1474.12\end{array}$ & $\begin{array}{r}0.34 \\
0.51 \\
3.75 \\
9.69 \\
21.76\end{array}$ & $\begin{array}{l}0.19 \\
0.1 \\
0.16 \\
0.24 \\
0.27\end{array}$ & $\begin{array}{l}0.22 \\
0.19 \\
0.86 \\
2.28 \\
5.96\end{array}$ \\
\hline \multicolumn{8}{|c|}{$\mathrm{Ar}$} \\
\hline $\begin{array}{l}0 \\
0.3 \\
0.5 \\
0.6 \\
0.71\end{array}$ & $\begin{array}{r}96.758 \\
239.766 \\
493.283 \\
766.364 \\
1399.43\end{array}$ & $\begin{array}{c}96.39 \\
236.757 \\
474.079 \\
703.433 \\
1021.53\end{array}$ & $\begin{array}{c}97.401 \\
241.35 \\
497.186 \\
772.878 \\
1410.92\end{array}$ & $\begin{array}{r}97.122 \\
239.073 \\
482.733 \\
726.082 \\
1152.17\end{array}$ & $\begin{array}{r}0.380 \\
1.255 \\
3.893 \\
8.212 \\
27.004\end{array}$ & $\begin{array}{l}0.661 \\
0.657 \\
0.785 \\
0.843 \\
0.814\end{array}$ & $\begin{array}{r}0.754 \\
0.969 \\
1.793 \\
3.119 \\
11.339\end{array}$ \\
\hline \multicolumn{8}{|c|}{$\mathrm{Kr}$} \\
\hline $\begin{array}{l}0 \\
0.3 \\
0.5 \\
0.6 \\
0.68\end{array}$ & $\begin{array}{l}73.152 \\
166.604 \\
313.05 \\
457.506 \\
656.201\end{array}$ & $\begin{array}{r}72.641 \\
163.271 \\
296.977 \\
413.889 \\
533.5\end{array}$ & $\begin{array}{r}73.967 \\
168.854 \\
318.69 \\
466.756 \\
670.137\end{array}$ & $\begin{array}{r}73.639 \\
166.722 \\
308.519 \\
439.682 \\
597.387\end{array}$ & $\begin{array}{r}0.699 \\
2.001 \\
5.134 \\
9.534 \\
18.699\end{array}$ & $\begin{array}{l}1.101 \\
1.333 \\
1.770 \\
1.982 \\
2.079\end{array}$ & $\begin{array}{r}1.355 \\
2.070 \\
3.741 \\
5.866 \\
10.694\end{array}$ \\
\hline \multicolumn{8}{|c|}{$\mathrm{Xe}$} \\
\hline $\begin{array}{l}0 \\
0.3 \\
0.4 \\
0.5 \\
0.6\end{array}$ & $\begin{array}{l}63.074 \\
133.725 \\
169.86 \\
218.946 \\
286.495\end{array}$ & $\begin{array}{l}62.823 \\
131.364 \\
167.74 \\
214.531 \\
271.761\end{array}$ & $\begin{array}{l}64.045 \\
135.78 \\
174.988 \\
227.526 \\
300.993\end{array}$ & $\begin{array}{r}63.895 \\
135.074 \\
173.734 \\
224.997 \\
293.426\end{array}$ & $\begin{array}{l}0.398 \\
1.766 \\
1.248 \\
2.017 \\
5.143\end{array}$ & $\begin{array}{l}1.516 \\
1.514 \\
2.930 \\
3.771 \\
4.817\end{array}$ & $\begin{array}{l}1.678 \\
2.747 \\
3.450 \\
4.651 \\
7.384\end{array}$ \\
\hline
\end{tabular}

Примечание. $\gamma_{1}=\left[\left|E_{\mathrm{zp}}\left(M T_{0}\right)-E_{\mathrm{zp}}\left(M T_{2}\right)\right| / E_{\mathrm{zp}}\left(M T_{0}\right)\right] \cdot 100 \%-$ вклад деформации электронных оболочек в парном и трехчастичном приближениях; $\gamma_{2}=\left[\left|E_{\mathrm{zp}}(M 3)-E_{\mathrm{zp}}\left(M T_{0}\right)\right| / E_{\mathrm{zp}}(M 3)\right] \cdot 100 \%$ - вклад трехчастичных сил связанных с перекрытием электронных оболочек; $\gamma_{3}=\left[\left|E_{\mathrm{zp}}(M 3 a)-E_{\mathrm{zp}}\left(M T_{2}\right)\right| / E_{\mathrm{zp}}(M 3 a)\right] \cdot 100 \%$ - вклад обоих видов трехчастичных сил, обязанных как перекрытию, так и деформации электронных оболочек.

для остальных КИГ. Средняя величина $\gamma_{1}$ для $\mathrm{Ar}$ растет по сравнению с $\mathrm{Ne}$ и составляет $21.76 \%$ при $u=0.71$, а для тяжелых КИГ падает и составляет $16.50 \%$ при $u=0.68$ для $\mathrm{Kr}$ и $11.53 \%$ при для $u=0.6 \mathrm{Xe}$.

Кроме расчетов в моделях $M T_{0}$ и $M T_{2}$ мы провели для всех КИГ расчеты фононных частот для двух точек главного значения в моделях M3 (парное взаимодействие в короткодействующем потенциале отталкивания без учета деформации электронных оболочек атома) и $M 3 a$ (парное взаимодействие и учет деформации электронных оболочек атома в парном приближении). Такое количество различных моделей необходимо для того, чтобы рассчитать вклады всех трехчастичных сил в фононные частоты. Из сравнения результатов расчета фононных частот в моделях $M 3$ и $M T_{0}$ можно сделать вывод, что относительный вклад трехчастичных сил за счет перекрытия электронных оболочек $\left(\gamma_{2}\right)$ по сравнению с парным достаточно маленький (сред- нее значение $\gamma_{2}$ для $\mathrm{Ne}$ равно $0.29 \%$ при $u=p=0$ и $0.53 \%$ при $u=0.76$; для $\operatorname{Ar} 1.68 \%$ при $u=0$ и $1.7 \%$ при $u=0.71$; для $\mathrm{Kr} 3.0 \%$ при $u=0$ и $4.13 \%$ при $u=0.68$; для Хе $4.69 \%$ при $u=0$ и 10.32\% при $u=0.6)$. Сравнение результатов в моделях $M 3 a$ и $M T_{2}$ показывает, что учет деформации электронных оболочек дипольного типа в трехчастичном приближении наряду с трехчастичными силами за счет перекрытия электронных оболочек приводит к увеличению вклада обоих видов трехчастичных сил в короткодействующем потенциале отталкивания при больших сжатиях (для $\mathrm{Ne}$ $\gamma_{3} \approx 5 \%$ при $u=0.76$, для Ar $\gamma_{3} \approx 10.07 \%$ при $u=0.71$, для $\mathrm{Kr} \gamma_{3} \approx 11.77 \%$ при $u=0.68$, для Хе $\gamma_{3} \approx 15.95 \%$ при $u=0.6)$. При $p=0$ среднее значение $\gamma_{3}$ близко к среднему значению $\gamma_{2}$ для всех КИГ. Отметим, что относительные вклады трехчастичного взаимодействия увеличиваются в ряду $\mathrm{Ne}-\mathrm{Xе.} \mathrm{Ограничение} \mathrm{сжатием}$ $u_{i}=0.76 ; 0.71 ; 0.68 ; 0.6$ связано с абсолютной неустой- 
чивостью ГЦК-решетки для $\mathrm{Ne}, \mathrm{Ar}, \mathrm{Kr}$ и Хе, соответственно [26].

\section{4. Энергия нулевых колебаний}

В гармоническом приближении энергия $E_{\mathrm{coh}}$ фононов задается известной формулой

$$
E_{\mathrm{coh}}=E_{\mathrm{zp}}+E^{*}=\frac{N_{A} \Omega}{(2 \pi)^{3}} \sum_{\lambda} \int d^{3} k \hbar \omega_{\lambda}(\mathbf{k})\left[\frac{1}{2}+n_{\lambda}(\mathbf{k})\right] .
$$

Здесь $n_{\lambda}(\mathbf{k})=\left[\exp \left(\hbar \omega_{\lambda}(\mathbf{k}) / k_{B} T\right)-1\right]^{-1}, E_{\text {zр }}-$ энергия нулевых колебаний (ей соответствует слагаемое $1 / 2$ в скобках в (13)), $N_{A}$ - число Авогадро, $\Omega=2 a^{3}-$ объем элементарной ячейки для КИГ в ГЦК-фазе, $a$ - параметр решетки, равный половине ребра куба, $k_{B}=1.3806662 \cdot 10^{-23} \mathrm{~J} / \mathrm{K}-$ постоянная Больцмана.

Для вычисления интегралов по зоне Бриллюэна будем использовать метод Чади-Коэна [23]. Сущность этого метода состоит в замене интеграла по зоне Бриллюэна суммой значений подынтегральной функции с соответствующими весами в особых точках (точках главного значения), найденных теоретико-групповыми методами [27].

В работе [23] авторы предложили метод генерирования этих точек на основе двух точек главного значения $\mathbf{k}_{1}$ и $\mathbf{k}_{2}$ для определения искомой функции $f(\mathbf{k})$ в кристалле

$$
\begin{gathered}
f(\mathbf{k})=\frac{1}{4}\left[3 f\left(\mathbf{k}_{1}\right)+f\left(\mathbf{k}_{2}\right)\right], \\
\mathbf{k}_{1}=\left[\frac{3}{4} ; \frac{1}{4} ; \frac{1}{4}\right], \quad \mathbf{k}_{2}=\left[\frac{1}{4} ; \frac{1}{4} ; \frac{1}{4}\right] .
\end{gathered}
$$

Тогда энергия нулевых колебаний, рассчитанная методом Чади-Коэна с использованием двухточечной схемы (14) будет иметь вид

$$
E_{\mathrm{zp}}=\frac{1}{2}\left[\frac{3}{4} \sum_{\lambda} \hbar \omega_{\lambda}\left(\mathbf{k}_{1}\right)+\frac{1}{4} \sum_{\lambda} \hbar \omega_{\lambda}\left(\mathbf{k}_{2}\right)\right] .
$$

На рисунке представлена энергия нулевых колебаний $E_{\text {zр }}$ кристаллов ряда $\mathrm{Ne}-$ Хе рассчитанная по двухточечной схеме Чади-Коэна (15) в моделях $M T_{2}$ и $M T_{0}$ при различных степенях сжатия. Видно, что наибольшее различие между моделями наблюдается для кристаллического $\mathrm{Ar}$ и проявляется при $u>0.5$ для $\mathrm{Ne}, \mathrm{Ar}$ и $\mathrm{Kr}$. Для Хе оно незначительно для всего интервала сжатий. Более детально вклады трехчастичных сил и деформации электронных оболочек в энергию нулевых колебаний показаны в табл. 2. Как видно с ростом давления (сжатия) наблюдается увеличение вклада деформации электронных оболочек дипольного типа в парном и трехчастичном приближении $\left(\gamma_{1}\right)$ в энергию нулевых колебаний. Из сравнения результатов расчета в моделях $M 3, M T_{0}\left(\gamma_{2}\right)$ и $M 3 a, M T_{2}\left(\gamma_{3}\right)$

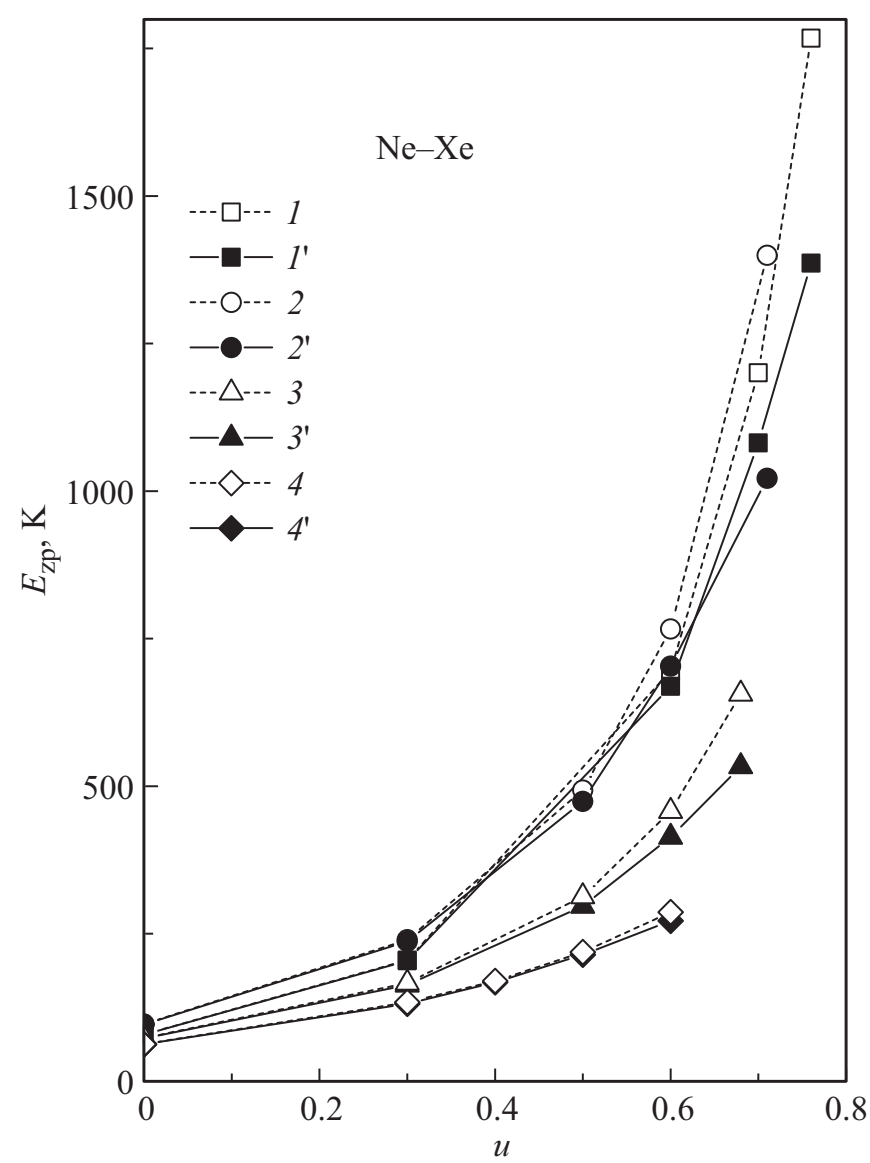

Зависимость энергии нулевых колебаний $E_{\text {zр }}$ от сжатия $u$ для $\mathrm{Ne}(1), \operatorname{Ar}(2), \operatorname{Kr}(3)$ и $\mathrm{Xe}(4) .1-4-E_{\text {zp }}$, рассчитанная в модели $M T_{0}$ (учитывается трехчастичное взаимодействие за счет перекрытия электронных оболочек и не учитывается деформация электронных оболочек атома); $1^{\prime}-4^{\prime}-$ в модели $M T_{2}$ (учитываются трехчастичные силы за счет перекрытия электронных оболочек и деформация электронных оболочек атомов дипольного типа в парном и трехчастичном приближениях).

видно, что относительные вклады трехчастичных сил за счет перекрытия электронных оболочек $\left(\gamma_{2}\right)$ малы и незначительно увеличиваются с ростом давления только для $\mathrm{Kr}$ и Хе. Влияние обоих видов трехчастичного взаимодействия на $E_{\text {zp }}$ более значительно $\gamma_{3}>\gamma_{2}$ для всего интервала сжатий, зависимость $\gamma_{3}(u)$ аналогична $\gamma_{1}(u)$. Для $E_{\text {zp }}$ сжатых КИГ тенденции поведения $\gamma_{i}$, ожидаемо, близки к поведению средних значений $\gamma_{i}$, полученных при расчете энергии фононных частот.

К сожалению, нам известны экспериментальные значения энергии нулевых колебаний $E_{\text {zp }}$ для $\mathrm{Ne}, \mathrm{Ar}$ и $\mathrm{Kr}$ только при $p=0[28,29]$. Рассчитанные нами величины $E_{\text {zp }}$ в модели $M T_{2}$ для $\mathrm{Ne}$ (см. табл. 2) $E_{\text {zp }}=79.185 \mathrm{~K}$. Экспериментальная энергия нулевых колебаний для $\mathrm{Ne} E_{\text {zp }}=78.5$ [28]. Погрешность наших расчетов по сравнению с экспериментальным значением составляет $\gamma=1.4 \%$. Экспериментальная энергия нулевых колебаний $E_{\mathrm{zp}}=86.1 \pm 2.5 \mathrm{~K}$ для $\mathrm{Ar}[29]$, и погрешность 
составляет $\gamma=11.95 \%, E_{\mathrm{zp}}=67.4 \pm 3 \mathrm{~K}$ для $\mathrm{Kr}[29]$ и погрешность составляет $\gamma=7.77 \%$. Хуже всего наша рассчитанная $E_{\text {zр }}$ согласуется с экспериментом для $\mathrm{Ar}$, хотя нужно учесть, что погрешность эксперимента составляет 3\% для $\mathrm{Ar}$ и 4\% для $\mathrm{Kr}$. Авторами работы [17] была рассчитана энергия нулевых колебаний для всего ряда $\mathrm{Ne}-Х е$ в модели Дебая по формуле $(9 / 8) k_{B} T_{\text {Debye }}[30]$ (температура Дебая была взята из работы [31]). Наименьшая погрешность с экспериментом получена для $\mathrm{Ne}\left(E_{\mathrm{zp}}=267 \mu\right.$ Hartree $(\mu \mathrm{H})=84.312 \mathrm{~K}$ $\left.\left(1 \mathrm{H}=2 \mathrm{Ry}=31.5777 \cdot 10^{4} \mathrm{~K}\right)\right)$ и составляет $\gamma \approx 7 \%$, то есть гораздо хуже согласуется с экспериментом, чем наши значения.

Отметим, что наилучшие значение $E_{z p}$ получены нами в модели $M T_{2}$, в которой учтены трехчастичное взаимодействие и деформация электронных оболочек атома дипольного типа в парном и трехчастичном приближениях.

\section{5. Заключение}

В работе [14], с использованием квантово-теоретического метода проводились исследования влияния парных, трехчастичных сил, эффектов энергии нулевых колебаний и ангармонизма на уравнение состояния сжатого $\mathrm{Ne}$. Количественный анализ показал, что наибольшее влияние, независимо от давления, имеют парные силы. Было также выявлено, что при высоком давлении индивидуальный вклад трехчастичных сил составил 33\%, а энергии нулевых колебаний, рассчитанной на основе модели Эйнштейна - 2\%. В то же время при низком давлении ситуация иная. Вклад трехчатичных сил составил $2.6 \%$, а нулевых колебаний - $11 \%$. Авторы [14], сравнивая результаты с экспериментальными значениями уравнения состояния, установили, что учет трехчастичного взаимодействие становится наиболее важным при высоких давлениях, а в области низкого давления доминируют энергии нулевых колебаний. Кроме этого, результаты [14] также подтверждают быструю сходимость разложения многочастичных сил для $\mathrm{Ne}$ даже при высоких давлениях. Поляризация $\mathrm{Ar}$ больше чем у $\mathrm{Ne}$, поэтому учет многочастичных сил становится более важным, сходимость $n$-частичного разложения несколько замедляется. Для получения хорошего согласия с экспериментом в расчет EOS Ar требуется включение, по крайней мере, четырехчастичных сил [15].

В работе [18] изучалось влияние на энергию нулевых колебаний трехчастичных сил и ангармонических эффектов в модели Эйнштейна для всего ряда КИГ при $p=0$. Рассматривалось 6 вариантов расчета для ГЦКи ГПУ-структур. Авторы не проводили сравнение с экспериментом. По нашему мнению наилучший результат для ГЦК- $\mathrm{Ne} E_{\mathrm{zp}}=240 \mu \mathrm{H}=75.79 \mathrm{~K}$ (погрешность с экспериментом $\gamma=3.1 \%$ ) получен в модели Эйнштейна при учете двух- и трехчастичных сил и полного учета ангармонических эффектов. Для остальных 5 вариантов $\gamma$ меняется от 4\% до 14\%. Для ГЦК-структуры
$\mathrm{Ar}$ и $\mathrm{Kr}$ лучшей моделью оказалась самая простая, в которой точно учитывается только двухчастичные силы $(\gamma=5.7 \%$ для $\mathrm{Ar}$ и $0.6 \%$ для $\mathrm{Kr})$. Учет трехчастичных сил и ангармонических эффектов приводит к увеличению погрешности для $\mathrm{Ar}$ до 13\%, а для $\mathrm{Kr}$ до 6\%.

В предыдущей работе [26] нами рассчитывались фононные частоты в симметричных направлениях волнового вектора $\mathbf{k}$ в широком интервале давлений для всего ряда кристаллов инертных газов. Было показано, что вклад трехчастичных сил за счет перекрытия электронных оболочек для КИГ мал по сравнению с парным взаимодействием как при низком, так и при высоком давлении. В то же время учет деформации электронных оболочек в парном и трехчастичном приближении приводит к размягчению „критических“ колебаний в определенных точках симметрии. Например, в $\mathrm{Ne}$ при сжатии $0.76(p=422 \mathrm{GPa})$ происходит размягчение продольной моды на границе зоны Бриллюэна в точках $X$ и $L$.

В настоящей работе мы исследовали фононные частоты в двух и десяти точках главного значения и влияние на них всех трехчастичных сил для сжатых кристаллов ряда $\mathrm{Ne}-\mathrm{Xe}$. Как и ранее, вклад трехчастичных сил за счет перекрытия электронных оболочек оказался мал на фоне парного взаимодействия даже при большом давлении (см. $\left.\gamma_{2}\right)$, а эффекты деформации электронных оболочек в парном и трехчастичном приближении $\left(\gamma_{1}\right)$ различны в разных точках главного значения. Как видно из табл. 1 вклад деформации электронных оболочек при сжатии $u=p=0$ варьируется от $0 \%$ до $0.39 \%$, а при $u=0.76$ изменяется от $0.35 \%$ до 55.9\%. При этом средняя величина вклада увеличивается с ростом давления от $0.15 \%$ до $17.2 \%$. Для энергии нулевых колебаний, представляющей собой интегральную функцию фононных частот, вклад деформации электронных оболочек в парном и трехчастичном приближении, ожидаемо, близок к средней величине вклада в фононных частотах. Для $\mathrm{Ne}$, например, $\gamma_{1}$ изменяется от $0.3 \%$ при $p=0$ до $22 \%$ при $u=0.76$ (см. табл. 2).

В классической версии теории Толпыго [32], параметры которой определялись из минимума среднеквадратичного отклонения для $\omega_{\lambda \mathbf{k}}$, при $p=0$ энергия нулевых колебаний для $\mathrm{Ne} E_{\mathrm{zp}}=79.2 \mathrm{~K}$, при этом погрешность с экспериментом составляет $\gamma=0.89 \%$. Для $\mathrm{Ar}$ и $\mathrm{Kr}$ $E_{\text {zp }}=91.4 \mathrm{~K} \quad(\gamma=6.16 \%)$ и $E_{\text {zp }}=69.5 \mathrm{~K} \quad(\gamma=3.1 \%)$, соответственно. Сравнение с нашими результатами в модели $M T_{2}$ дает следующие погрешности: для $\mathrm{Ne}$ $\gamma=0.02 \%$, для $\operatorname{Ar} \gamma=5.4 \%$, для $\mathrm{Kr} \gamma=4.5 \%$ и для Хе $\gamma=0.12 \%$.

Таким образом, полученные нами результаты расчета $E_{\mathrm{zp}}$ с учетом трехчастичных сил и деформации электронных оболочек атомов дипольного типа в парном и трехчастичном приближении удовлетворительно согласуются с экспериментом и очень близки к результатам расчета в классической модели Толпыго. 


\section{Конфликт интересов}

Авторы заявляют, что у них нет конфликта интересов.

\section{Список литературы}

[1] T. Kihara, S. Koba. J. Phys. Soc. Jpn. 7, 348 (1952).

[2] M. Born. Proc. Cambridge Philos. Soc. 40, 262 (1944).

[3] J.A. Prins, J.M. Dumore, L.T. Tjoan. Physica (Amsterdam) 18, 307 (1952).

[4] G.L. Pollack. Rev. Mod. Phys. 36, 748 (1964).

[5] B.W. van de Waal. Phys. Rev. Lett. 67, 3263 (1991).

[6] J.A. Venables. In: Rare Gas Solids/ Ed. M.L. Klein, J.A. Venables. Academic Press, London (1976).

[7] E. Kim, M. Nicol. Phys. Rev. Lett. 96, 035504 (2006).

[8] I. Kwon, L.A. Collins, J.D. Kress, N. Troullier. Phys. Rev. B 52, 21, 15165 (1995).

[9] J.K. Dewhurst, R. Ahuja, S. Li, B. Johansson. Phys. Rev. Lett. 88, 7, 075504 (2002).

[10] M.G. Medvedev, I.S. Bushmarinov, J. Sun, J.P. Perdew, K.A. Lyssenko. Science 355, 49 (2017).

[11] H. Larsen, J. Olsen, C. Hattig, P. Jorgensen, O. Christiansen, J. Gauss. J. Chem. Phys. 111, 1917 (1999).

[12] N. Gaston, P. Schwerdtfeger. Phys. Rev. B 74, 024105 (2006).

[13] F.O. Kannemann, A.D. Becke. J. Chem. Theory Comput. 5, 719 (2009).

[14] P. Schwerdtfeger, A. Hermann. Phys. Rev. B 80, 064106 (2009).

[15] P. Schwerdtfeger, K.G. Steenbergen, E. Pahl. Phys. Rev. B 95, 214116 (2017).

[16] K. Rosciszewski, B. Paulus, P. Fulde, H. Stoll. Phys. Rev. B 62, 5482 (2000).

[17] K. Rosciszewski, B. Paulus, P. Fulde, H. Stoll. Phys. Rev. B 60, 7905 (1999).

[18] K. Rosciszewski, B. Paulus. Phys. Rev. B 66, 092102 (2002).

[19] L. Trombach, R.S. Hoy, D.J. Wales, P. Schwerdtfeger. Phys. Rev. E 97, 043309 (2018).

[20] L. Trombach, P. Schwerdtfeger. Phys. Rev. E 98, 033311 (2018).

[21] Е.П. Троицкая, Е.Е. Горбенко, Е.А. Пилипенко. ФНТ 42, 526 (2016).

[22] Е.П. Троицкая, В.В. Чабаненко, Е.Е. Горбенко, Е.А. Пилипенко. ФТТ 57, 114 (2015).

[23] D.J. Chadi, M.L. Cohen. Phys. Rev. B 8, 5747 (1973).

[24] Е.А. Пилипенко, Е.П. Троицкая, Е.Е. Горбенко. ФТВД 29, 1, 64 (2019).

[25] Е.П. Троицкая, В.В. Чабаненко, Е.Е. Горбенко, Н.В. Кузовой. ФТВД 17, 3, 14 (2007).

[26] Е.П. Троицкая, Е.А. Пилипенко, Е.Е. Горбенко. ФТТ 61, 154 (2019).

[27] A. Baldereschi. Phys. Rev. B 7, 5212 (1973).

[28] J. Eckert, W.B. Daniels, J.D. Axe. Phys. Rev. B 14, 8, 3649 (1976).

[29] R.H. Beaumont, H. Chihara, J.A. Morrison. Proc. Phys. Soc. 78, 506, 1462 (1961).

[30] B. Farid, R. Godby. Phys. Rev. B 43, 14248 (1991).

[31] Data compiled by N. Pearlman. American Institute of Physics. Handbook. 3rd ed./ Ed. Dwight E. Gray (Mc Graw-Hill, N. Y., 1965).

[32] Е.В. Зароченцев, К.Б. Толпыго, Е.П. Троицкая. ФНТ 5, 11, 1324 (1979).

Редактор Ю.Э. Китаев 